\title{
EXTENDING MULTIPLIERS FROM SEMIGROUPS
}

\author{
MARCELO LACA AND IAIN RAEBURN
}

(Communicated by Palle E. T. Jorgensen)

\begin{abstract}
A multiplier on a normal subsemigroup of a group can be extended to a multiplier on the group. This is used to show that normal cancellative semigroups have the same second cohomology as the group they generate, generalising earlier results of Arveson, Chernoff, and Dinh. The main tool is a dilation theorem for isometric multiplier representations of semigroups.
\end{abstract}

\section{INTRODUCTION}

A subsemigroup $S$ of a group $G$ is a nonempty subset closed under the group operation. We assume $x S x^{-1} \subset S$ for every $x \in G$, and say the semigroup is normal; in order to avoid trivialities, we also assume that $S$ generates $G$ as a group. A multiplier on $S$ is a function $\sigma$ from $S \times S$ into the circle group $\mathbb{T}$ such that

$$
\sigma(x, y) \sigma(x y, z)=\sigma(x, y z) \sigma(y, z) \quad(x, y, z \in S) .
$$

The purpose of this note is to show that every multiplier on a normal subsemigroup $S$ extends to a multiplier on all of $G$. This generalizes a result of Arveson [Arv] for $S=[0, \infty)$, which was subsequently extended by Chernoff in [Che] to $S=[\alpha, \infty)$, and work of Hung Dinh, who proved in [Din] that multipliers of any discrete subsemigroup of $\mathbb{R}^{+}$extend to multipliers of the corresponding subgroup. Our hypothesis of normality is satisfied by any subsemigroup obtained by pulling back a generating subsemigroup from an abelian quotient. In particular, it includes the subsemigroups considered in [P-R], which are pulled back from the positive cone in a totally ordered quotient.

In $\S 1$ we prove that homomorphisms on normal subsemigroups extend uniquely to the group generated, and make a few comments about normal semigroups, which may be ignored by those primarily interested in positive cones of ordered groups. In $\S 2$ we generalise a dilation theorem of Phillips and Raeburn [P-R], which is a multiplier version of a theorem of Douglas [Dou], and use it to extend multipliers from subsemigroups of discrete groups. Subsemigroups of

Received by the editors May 5, 1993.

1991 Mathematics Subject Classification. Primary 20C25, 47A20; Secondary 46L55, 47D03.

Key words and phrases. Semigroup multiplier, isometric multiplier representations, second cohomology.

This research was supported by the Australian Research Council. 
locally compact groups are discussed in $\S 3$, where, assuming that $S$ has nonzero Haar measure, we show that the extension result holds for Borel multipliers on normal subsemigroups of second countable locally compact groups.

The authors thank Dana Williams for several useful comments, and George Willis for suggesting Example 1.3.

\section{NORMAL SUBSEMIGROUPS}

We begin by observing that if $S$ is a normal generating subsemigroup of $G$, then $G$ coincides with the set $S S^{-1}=\left\{s t^{-1}: s, t \in S\right\}$; for if $s, t, x, y \in S$, then $s t^{-1} x y^{-1}=s\left(t^{-1} x t\right)(y t)^{-1}$, so $S S^{-1}$ is a subgroup of $G$ containing $S$, hence equal to $G$. In the motivating examples, where $S$ is the positive cone of a totally ordered group, $G$ is actually $S \cup S^{-1}$, but the subsemigroups $S=[\alpha, \infty)$ of $\mathbb{R}$ considered in [Che] and the positive cones of partially ordered abelian groups provide examples in which $S \cup S^{-1}$ is strictly smaller than $G$.

The following property of normal, generating subsemigroups is essential to our approach. It is probably well known to semigroup theorists, but we have been unable to find a suitable reference.

Lemma 1.1. Suppose $S$ is a normal subsemigroup generating the group $G$, and let $\phi$ be a homomorphism from $S$ into a group. Then there exists a unique group-homomorphism $\varphi$ extending $\phi$ to all of $G$.

Proof. Since every element of $G$ has the form $s t^{-1}$ with $s$ and $t$ in $S$, it suffices to show that the map $\varphi: s t^{-1} \mapsto \phi(s) \phi(t)^{-1}$ is a well-defined grouphomomorphism on all of $G$.

Note that for $y, t \in S$,

$$
\phi\left(y^{-1} t y\right)=\phi(y)^{-1} \phi(y) \phi\left(y^{-1} t y\right)=\phi(y)^{-1} \phi(t) \phi(y) \text {. }
$$

Suppose now $x y^{-1}=s t^{-1}$ for $x, y, s, t \in S$; then $x y^{-1} t y=s y$ and (1.1) implies

$$
\phi(x) \phi(y)^{-1} \phi(t) \phi(y)=\phi(x) \phi\left(y^{-1} t y\right)=\phi\left(x y^{-1} t y\right)=\phi(s) \phi(y) .
$$

Since $\phi$ takes values on a group, this implies $\phi(x) \phi(y)^{-1}=\phi(s) \phi(t)^{-1}$, so $\varphi$ is well defined. On the other hand,

$$
\begin{aligned}
\varphi\left(x y^{-1} s t^{-1}\right) & =\varphi\left(x y^{-1} s y y^{-1} t^{-1}\right)=\varphi\left(x y^{-1} s y(t y)^{-1}\right)=\phi\left(x y^{-1} s y\right) \phi(t y)^{-1} \\
& =\phi(x) \phi(y)^{-1} \phi(s) \phi(y) \phi(y)^{-1} \phi(t)^{-1}=\varphi\left(x y^{-1}\right) \varphi\left(s t^{-1}\right),
\end{aligned}
$$

so $\varphi$ is a homomorphism on $G$.

Remark 1.2. (i) Normal generating subsemigroups can be characterized intrinsically as the cancellative semigroups $S$ for which $S s=s S$ for all $s \in S$. For if $S$ is such a semigroup, then $s t \in S s \cap S t$ for $s, t \in S$, and Ore's condition of right reversibility holds (see, e.g., [C-P, $\S 1.10]$ ); thus by [C-P, Theorem 1.23] and Lemma 1.1, $S$ uniquely determines a group $G$ in which it embeds as a generating subsemigroup. The set $\{x \in G: x S=S x\}$ is a subgroup of $G$ which contains $S$ by assumption, so $S$ embeds as a normal subsemigroup of $G$. 
(ii) Although we shall not explicitly use it later, it is worth noting here that a normal subsemigroup makes the group it generates into a directed set. In the case of a subsemigroup of a discrete abelian group, this fact was observed and used by Douglas in his proof of Ito's dilation theorem [Dou], which motivated [P-R, Theorem 2.1] and our Theorem 2.1. Suppose $S$ is a normal generating subsemigroup of $G$, and define $x \succ y$ for $x, y \in G$ to mean $x \in S y$; transitivity follows from the semigroup property, and cofinality of $\succ$ is a consequence of normality because $s t=\left(s t s^{-1}\right) s \in S s \cap S t$ whenever $s, t \in S$; in particular $S s \cap S t$ is never empty. The relation $\succ$ need not be reflexive or antisymmetric, but is nevertheless a preorder compatible with the group structure in the sense that $x \succ y$ and $z \in S$ imply $x z \succ y z$. Conversely, if $\succ$ is a cofinal transitive relation in $G$ satisfying this last property, then $\{x \in G: x \succ e\}$ is a normal generating subsemigroup of $G$.

Example 1.3. Normal subsemigroups are more general than the semigroups pulled back from abelian quotients. For example, consider the semigroup

$$
S=\{(a, b): \text { either } a>1 \text { and } b \in \mathbb{R} \text {, or } a=1 \text { and } b>0\}
$$

of the ' $a x+b$ group' $G=(0, \infty) \rtimes \mathbb{R}$. Calculations show that $S$ is both normal and generating. It turns out that if $N$ is any normal subgroup of $G$ with $G / N$ abelian, then $N$ must contain $R=\{(1, b): b \in \mathbb{R}\}$, so $G / N \cong$ $(G / R) /(N / R)$ is a quotient of $(0, \infty)$. But $S$ is not the inverse image of any set in any quotient of $(0, \infty)$. This construction generalizes to semidirect products of groups having normal generating subsemigroups compatible with the action.

\section{ISOMETRIC MULTIPLIER REPRESENTATIONS}

A family of isometries $\left\{V_{s}: s \in S\right\}$ satisfying

$$
V_{s} V_{t}=\sigma(s, t) V_{s t} \quad \text { for } s, t \in S,
$$

where $\sigma$ is a multiplier on $S$, is called an isometric $\sigma$-representation of $S$. Isometric multiplier representations exist for every multiplier, as the following shows.

If $\sigma$ is a multiplier on $S$, define the left regular $\sigma$-representation of $S$ on $\ell^{2}(S)$ by

$$
\left(L_{s}^{\sigma} f\right)(t)= \begin{cases}\sigma\left(s, s^{-1} t\right) f\left(s^{-1} t\right) & \text { if } t \in s S, \\ 0 & \text { otherwise }\end{cases}
$$

for $f \in \ell^{2}(S)$ and $s \in S$. The family $\left\{L_{s}^{\sigma}: s \in S\right\}$ is an isometric $\sigma$ representation of $S$ consisting of nonunitary isometries.

The following theorem shows that an isometric $\sigma$-representation of $S$ is always the restriction of a $\sigma$-representation of $S$ by unitary operators to an invariant subspace.

Theorem 2.1. Suppose $\sigma$ is a multiplier on the normal generating subsemigroup $S$ of the group $G$, and let $\left\{V_{s}: s \in S\right\}$ be an isometric $\sigma$-representation of $S$ on a Hilbert space $H$. Then there exists a unitary $\sigma$-representation of $S$ on a Hilbert space $\mathscr{H}$ containing a copy of $H$ such that

(i) $U_{s}$ leaves $H$ invariant and $\left.U_{s}\right|_{H}=V_{s}$; and

(ii) $\bigcup_{s \in S} U_{s}^{*} H$ is dense in $\mathscr{H}$. 
Proof. The proof follows the construction in [P-R, Theorem 2.1], except that here $\sigma$ need only be defined on $S$. Let $H_{0}$ be the set of functions $f: S \rightarrow H$ for which there exists $s \in S$ such that

$$
f(y)=\overline{\sigma\left(y s^{-1}, s\right)} V_{y s^{-1}}(f(s)) \quad \text { for } y \in S s .
$$

Such $s$ will be called admissible for $f$. Note that if $s$ is admissible for $f$ and $r \in S s$, then $r$ is also admissible for $f$, for then $S r \subset S s$, and for all $y \in S r$,

$$
\begin{aligned}
f(y) & =\overline{\sigma\left(y s^{-1}, s\right)} V_{y s^{-1}} f(s)=\overline{\sigma\left(y s^{-1}, s\right)} \overline{\sigma\left(y r^{-1}, r s^{-1}\right)} V_{y r^{-1}} V_{r s^{-1}} f(s) \\
& =\overline{\sigma\left(y s^{-1}, s\right)} \overline{\sigma\left(y r^{-1}, r s^{-1}\right)} \sigma\left(r s^{-1}, s\right) V_{y r^{-1}} f(r) \\
& =\overline{\sigma\left(y r^{-1}, r\right)} V_{y r^{-1}} f(r) .
\end{aligned}
$$

Suppose now $f$ and $g$ are in $H_{0}$, and $s$ is admissible for both $f$ and $g$ (since $S$ is normal, the product of an admissible value for $f$ and one for $g$ will do). If $y \in S s$, then

$$
\langle f(y), g(y)\rangle=\left\langle\overline{\sigma\left(y s^{-1}, s\right)} V_{y s^{-1}} f(s), \overline{\sigma\left(y s^{-1}, s\right)} V_{y s^{-1}} g(s)\right\rangle=\langle f(s), g(s)\rangle,
$$

because $V_{y s^{-1}}$ is an isometry and $\sigma$ takes values on the unit circle. Thus $\langle f(s), g(s)\rangle$ is constant on the set of values of $s$ which are admissible for both functions, and we can define a positive semidefinite sesquilinear functional on $H_{0}$ by

$$
\langle f, g\rangle=\langle f(s), g(s)\rangle,
$$

where $s$ is any value admissible for both $f$ and $g$.

Let $\mathscr{H}$ be the Hilbert space completion of $H_{0}$ under the corresponding seminorm, and notice that this identifies functions which coincide on an admissible set of the form $S s$. To embed the original Hilbert space $H$, define, for each $\xi \in H$, the function $\widehat{\xi}$ by $\widehat{\xi}(s)=V_{s} \xi$ for $s \in S$. Since $V$ is an isometric $\sigma$ representation, $\widehat{\xi}$ satisfies (2.1) for any $s \in S$, hence $\widehat{\xi} \in H_{0}$ and every $s \in S$ is admissible for $\widehat{\xi}$. The embedding $\xi \mapsto \widehat{\xi}$ is isometric because each $V_{s}$ is.

Suppose now that $f \in H_{0}$ and $t \in S$, and consider the function $f_{t}$ defined by $f_{t}(x)=\sigma(x, t) f(x t)$ for $x \in S$. If $s \in S$ is admissible for $f$, then normality implies that $s t$ is also admissible for $f$, and since $x t \in S s t$ for any $x \in S s$,

$$
\begin{aligned}
\sigma(x, t) f(x t) & =\sigma(x, t) \overline{\sigma\left(x t(s t)^{-1}, s t\right)} V_{x t(s t)^{-1}} f(s t) \\
& =\sigma(x, t) \overline{\sigma\left(x s^{-1}, s t\right)} V_{x s^{-1}} f(s t) \\
& =\overline{\sigma\left(x s^{-1}, s\right)} V_{x s^{-1}}(\sigma(s, t) f(s t))
\end{aligned}
$$

which shows that the same $s$ is admissible for $f_{t}$; in particular, $f_{t} \in H_{0}$.

Evaluating the inner product at a point $s$ admissible for both $f$ and $g$, we obtain $\left\langle f_{t}, g_{t}\right\rangle=\left\langle f_{t}(s), g_{t}(s)\right\rangle=\langle\sigma(s, t) f(s t), \sigma(s, t) g(s t)\rangle=\langle f, g\rangle$; thus, $U_{t} f=f_{t}$ for $t \in S$ defines an isometry $U_{t}$ on $\mathscr{H}$.

If $\xi \in H$, then

$$
\left(U_{t} \widehat{\xi}\right)(x)=\sigma(x, t) V_{x t} \xi=V_{x} V_{t} \xi=\widehat{V_{t} \xi}(x) \quad \text { for } x \in S,
$$


so $U_{t}$ restricts to $V_{t}$ on the copy of $H$ inside $\mathscr{H}$. Furthermore,

$$
\begin{aligned}
U_{s} U_{t} f(x) & =\sigma(x, s) U_{t} f(x s)=\sigma(x, s) \sigma(x s, t) f(x s t)=\sigma(x, s t) \sigma(s, t) f(x s t) \\
& =\sigma(s, t) U_{s t} f(x) \quad \text { for all } x \in S \text { and } f \in H_{0} .
\end{aligned}
$$

Thus $U$ is a $\sigma$-representation of $S$ by isometries, and it remains to prove that these isometries are in fact unitaries. Let $t \in S$, and suppose that $S$ is admissible for $g \in H_{0}$. Consider the function defined by

$$
g_{t^{-1}}(x)= \begin{cases}\overline{\sigma\left(x t^{-1}, t\right)} g\left(x t^{-1}\right) & \text { if } x \in S t \\ 0 & \text { otherwise. }\end{cases}
$$

Then $s t$ is admissible for $g_{t^{-1}}$ : if $x \in S s t$, then $x t^{-1} \in S s$ is admissible for $g$, and

$$
\begin{aligned}
g_{t^{-1}}(x) & =\overline{\sigma\left(x t^{-1}, t\right)} g\left(x t^{-1}\right) \\
& =\overline{\sigma\left(x t^{-1}, t\right)} \overline{\sigma\left(x t^{-1} s^{-1}, s\right)} V_{x t^{-1} s^{-1}} g(s) \\
& =\overline{\sigma\left(x t^{-1} s^{-1}, s t\right)} \overline{\sigma(s, t)} V_{x t^{-1} s^{-1}} g(s) \\
& =\overline{\sigma\left(x(s t)^{-1}, s t\right)} \frac{\sigma\left(s t t^{-1}, t\right)}{\sigma x(s t)^{-1}} g\left(s t t^{-1}\right) \\
& =\overline{\sigma\left(x(s t)^{-1}, s t\right)} V_{x(s t)^{-1}} g_{t^{-1}}(s t)
\end{aligned}
$$

which puts $g_{t^{-1}}$ in $H_{0}$. Since

$$
U_{t} g_{t^{-1}}(x)=\sigma(x, t) g_{t^{-1}}(x t)=\sigma(x, t) \overline{\sigma\left(x t t^{-1}, t\right)} g\left(x t t^{-1}\right)=g(x)
$$

for $x \in S, U_{t}$ is surjective for every $t \in S$. Thus $U$ is a unitary $\sigma$ representation of the subsemigroup $S$ on $\mathscr{H}$, which finishes the proof of (i).

To prove (ii), assume $f \in H_{0}$ and fix $s$ admissible for $f$. Then for $x \in S s$,

$$
U_{s}(f)(x)=\sigma(x, s) f(x s)=\sigma(x, s) \overline{\sigma\left(x s s^{-1}, s\right)} V_{x s s^{-1}} f(s)=V_{x}(f(s))=\widehat{f(s)}(x) .
$$

Hence $f(x)=\left(U_{s}^{*} \widehat{f(s)}\right)(x)$ for $x$ in the admissible set $S s$, which implies $f=U_{s}^{*} \widehat{f(s)}$ in $\mathscr{H}$. Since $H_{0}$ is dense in $\mathscr{H}$, (ii) follows.

Theorem 2.2. Let $S$ be a normal generating subsemigroup of the group $G$. Every multiplier on $S$ extends to a multiplier on $G$.

Proof. Suppose $\sigma$ is a multiplier on $S$, and let $V$ be an isometric $\sigma$-representation of $S$ (e.g., the left regular $\sigma$-representation). Let $U$ be the unitary dilation given by Theorem 2.1.

For each $x \in G$ let $s_{x}$ and $t_{x}$ be elements of $S$ such that $x=s_{x} t_{x}^{-1}$, and define

$$
W_{x}= \begin{cases}\sigma\left(x, t_{x}\right) U_{s_{x}} U_{t_{x}}^{*} & \text { if } x \in S, \\ U_{s_{x}} U_{t_{x}}^{*} & \text { otherwise. }\end{cases}
$$

If $\pi: U(\mathscr{H}) \rightarrow P U(\mathscr{H})$ is the quotient map from unitaries on $\mathscr{H}$ onto the projective unitaries on $\mathscr{H}$, then $s \in S \mapsto \pi U_{s} \in P U(\mathscr{H})$ is a semigrouphomomorphism, and since $\pi W_{x}=\pi U_{s_{x}}\left(\pi U_{t_{x}}\right)^{-1}$, Lemma 1.1 implies that $\pi W$ is a group homomorphism (the unique extension of $\pi U$ ). Thus $W$ is a multiplier representation of $G$ with multiplier $\omega$ characterized by $W_{x} W_{y} W_{x y}^{*}=$ $\omega(x, y) I$. 
If $x \in S$, then $x t_{x}=s_{x}$ implies that $U_{x} U_{t_{x}}=\sigma\left(x, t_{x}\right) U_{s_{x}}$, and hence that $W_{x}=U_{x}$. Therefore, $W$ extends $U$ to all of $G$, and $\omega$ coincides with $\sigma$ on $S$.

Although it is possible to write down explicit formulas for the extension of $\sigma$ once the extension $W$ of the $\sigma$-representation $U$ has been defined, we have chosen the indirect approach of projective representations to avoid the lengthy case-by-case consideration needed to verify that those formulas actually define a multiplier. Similar comments apply to the proof of the following corollary. As a bonus, our approach yields a direct generalisation of [P-R, Theorem 2.1], Corollary 2.4 below, which is potentially of independent interest.

The second cohomology group of a semigroup is the quotient of the group $Z^{2}(S, \mathbb{T})$ of all multipliers by the subgroup $B^{2}(S, \mathbb{T})$ of coboundaries, i.e.,

$$
H^{2}(S, \mathbb{T})=Z^{2}(S, \mathbb{T}) / B^{2}(S, \mathbb{T})
$$

Corollary 2.3. Restriction of multipliers on $G$ to multipliers on $S$ induces a natural isomorphism of $H^{2}(G, \mathbb{T})$ onto $H^{2}(S, \mathbb{T})$.

Proof. Restriction is clearly a homomorphism from $Z^{2}(G, \mathbb{T})$ into $Z^{2}(S, \mathbb{T})$ and is surjective by Theorem 2.2 .

Suppose $\sigma$ is a multiplier on $G$ whose restriction to $S$ is trivial, so there is a function $\lambda: S \rightarrow \mathbb{T}$ such that $\sigma(s, t)=\lambda(s t) \overline{\lambda(s) \lambda(t)}$ for $s, t \in S$. If $U$ is a unitary $\sigma$-representation of $G$, then $s \in S \mapsto \lambda(s) U_{s}$ is a unitary representation of $S$. Let $W$ denote its unique extension to all of $G$, which exists by Lemma 1.1, and observe that $\pi U$ coincides with $\pi W$ on $S$, where $\pi$ is the quotient map in the proof of Theorem 2.2. From the uniqueness part of Lemma 1.1 they must coincide on all of $G$. Therefore, $U_{x} W_{x}^{*}$ is a scalar for each $x \in G$, so $U_{x}=\mu(x) W_{x}$, for some function $\mu: G \rightarrow \mathbb{T}$. Since $W$ is a true representation, the multiplier $\sigma$ of $U$ must equal $\partial \mu$. The restriction map is thus one-to-one modulo coboundaries.

Corollary 2.4. Suppose $V$ is a $\sigma$-representation of $S$ as in Theorem 2.1. If $\rho$ is a multiplier on $G$ extending $\sigma$, then there exists a unitary $\rho$-representation $R$ of the group $G$ on $\mathscr{H}$ such that $\left.R_{S}\right|_{H}=V_{s}$ for $s \in S$.

Proof. Let $W$ be the unitary $\omega$-representation of $G$ constructed in the proof of Theorem 2.2. Since $\rho$ is an extension of $\sigma$, it must be cohomologous to the multiplier $\omega$ of $W$, so it suffices to multiply $W$ by a convenient scalar function to obtain a $\rho$-representation of $G$ dilating $V$.

\section{SUBSEMIGROUPS OF LOCALLY COMPACT GROUPS}

Throughout this section $G$ denotes a second countable locally compact group, and $S$ a normal generating subsemigroup which is a Borel subset of $G$. In order to extend a Borel measurable multiplier from $S$ to $G$, we will have to assume that $S$ is not a null set; it is possible that normal generating Borel subsemigroups are necessarily nonnull, but we do not know how to prove it.

Lemma 3.1. The following are equivalent.

(i) $S$ has nonzero Haar measure.

(ii) $S$ has nonempty interior.

(iii) $S$ contains a cofinal sequence, i.e., a sequence $\left\{s_{n}\right\}$ with $\bigcup_{n} s_{n} S^{-1}=G$. 
Proof. If $S$ is nonnull, then it contains a set $A$ of nonzero finite measure. The function $1_{A} * 1_{A} \in L^{1}(G)$ is continuous, has support in $A^{2} \subset S$, and is not identically zero because $\left\|1_{A} * 1_{A}\right\|_{1}=\int_{G} \int_{G} 1_{A}(x) 1_{A}\left(x^{-1} y\right) d x d y=\mu(A)^{2}$. This proves that $S$ has nonempty interior.

Suppose now the interior of $S$ is not empty. Let $\left\{x_{n}\right\}$ be a dense sequence in $G$, which exists by second countability. For each $x \in G$ the set $x S$ has nonempty interior and, therefore, $x_{n} \in x S$ for some $n$; this shows that $\left\{x_{n}\right\}$ is cofinal. If necessary, substitute $x_{n}$ by any $s_{n} \in x_{n} S \cap S$ to obtain a cofinal sequence which lies in $S$.

If $\left\{s_{n}\right\}$ is a cofinal sequence, then for some $n \in \mathbb{N}$ the set $s_{n} S^{-1}$ has nonzero measure because $\bigcup_{n} s_{n} S^{-1}=G$; therefore, $S$ has nonzero measure.

Assume from now on that $S$ is not a null set, and let $\left\{s_{n}\right\}$ be a cofinal sequence in $S$; for each $x \in G$ define $s_{x}$ to be $s_{n}$ where $n$ is the smallest positive integer for which $x \in s_{n} S^{-1}$. This defines a Borel function on all of $G$ because $\left\{x: s_{x}=s_{n}\right\}=s_{n} S^{-1} \backslash \cup_{k<n}\left(s_{k} S^{-1}\right)$ is a Borel set for each $n$. Let $t_{x}=x^{-1} s_{x}$ to obtain a Borel map $x \mapsto\left(s_{x}, t_{x}\right)$ from $G$ into $S \times S$ such that $x=s_{x} t_{x}^{-1}$. The immediate consequence is that Lemma 1.1 holds for a Borel homomorphism of $S$ into a topological group, giving a unique Borel group-homomorphism extension.

The appropriate measurability condition for isometric multiplier representations corresponding to Borel multipliers is that of weak Borel measurability, i.e., that the maps $s \mapsto\left\langle V_{s} \xi, \eta\right\rangle$ are Borel maps for all $\xi, \eta \in H$. If the Hilbert space $H$ is separable, this coincides with strong Borel measurability (if $\left\{e_{n}\right\}$ is an orthonormal basis, then $\left.V_{s} \xi=\sum_{n}\left\langle V_{s} \xi, e_{n}\right\rangle e_{n}\right)$.

The left regular $\sigma$-representation of $S$ is defined on the (nontrivial) subspace $L^{2}(S)$ of $L^{2}(G)$ consisting of functions vanishing almost everywhere outside $S$. For each $s \in S$,

$$
\left(L_{s}^{\sigma} f\right)(t)= \begin{cases}\sigma\left(s, s^{-1} t\right) f\left(s^{-1} t\right) & \text { if } t \in s S \\ 0 & \text { otherwise }\end{cases}
$$

gives a $\sigma$-representation of $S$ as isometries on $L^{2}(S)$. To verify that the correspondence $s \mapsto L_{s}^{\sigma}$ is weakly Borel, consider functions $f$ and $g$ in $L^{2}(S)$ : the map

$$
s \mapsto\left\langle L_{s}^{\sigma} f, g\right\rangle=\int_{s S} \sigma\left(s, s^{-1} t\right) f\left(s^{-1} t\right) \overline{g(t)} d t=\int_{S} \sigma(s, u) f(u) \overline{g(s u)} d u
$$

is Borel because the integrand can be written as a linear combination of four nonnegative Borel functions to which Tonelli's theorem applies.

Theorem 3.2. Suppose $\sigma$ is a Borel multiplier on $S$, and let $\left\{V_{s}: s \in S\right\}$ be a Borel isometric $\sigma$-representation of $S$ on a separable Hilbert space $H$. Then there exists a Borel unitary $\sigma$-representation $U$ of $S$ on a separable Hilbert space $\mathscr{H}$ containing a copy of $H$ such that

(i) $U_{s}$ leaves $H$ invariant and $\left.U_{s}\right|_{H}=V_{s}$; and

(ii) $\bigcup_{s \in S} U_{s}^{*} H$ is dense in $\mathscr{H}$.

Proof. The proof of Theorem 2.1 carries over to the present situation. Since $V$ is strongly Borel measurable, the admissibility condition (2.1) shows that any $f \in H_{0}$ coincides with a Borel function from $S$ to $H$ on a set of the form $S s$, 
proving that the Borel functions in $H_{0}$ give a dense set in the completion $\mathscr{H}$. In order to prove that the map $t \mapsto U_{t}$ is weakly Borel, take Borel functions $f, g \in H_{0}$ and let $s \in S$ be a fixed admissible value for both. The formula $\left\langle U_{t} f, g\right\rangle=\left\langle f_{t}(s), g(s)\right\rangle=\langle\sigma(s, t) f(s t), g(s)\rangle$ defines a Borel function of $t$. Since Borel functions in $H_{0}$ are dense in $\mathscr{H}$, the Borel measurability of $U$ follows.

It only remains to check that $\mathscr{H}$ is separable. By the cofinality of $\left\{s_{n}\right\}$, which is equivalent to $\cup_{n} S^{-1} s_{n}=G$ because $S$ is normal, for each $s \in S$ there exists $n \in \mathbb{N}$ and $t \in S$ with $s_{n}=t s$, hence $U_{t} U_{s}=\sigma(t, s) U_{s_{n}}$. Thus $U_{s}^{*} H=U_{s_{n}}^{*} U_{t} H$, and since $U_{t}$ leaves $H$ invariant, the union in (ii) can be taken over the cofinal sequence $\left\{s_{n}\right\}$ without altering the result; the separability of $H$ then implies that of $\mathscr{H}$.

Using the Borel version of Lemma 1.1, and Theorem 3.2 in place of Theorem 2.1, we can extend Borel multipliers on $S$ and deduce the same corollaries.

Theorem 3.3. Every Borel multiplier on $S$ extends to a Borel multiplier on $G$. Proof. Theorem 3.2 applies to the left regular $\sigma$-representation of $S$, giving a minimal unitary dilation on a separable Hilbert space $\mathscr{H}$. Since there exists a Borel map $x \mapsto\left(s_{x}, t_{x}\right)$ with $x=s_{x} t_{x}^{-1}$, the same argument used to prove Theorem 2.2 can be used here.

Corollary 3.4. Restriction of Borel multipliers on $G$ to Borel multipliers on $S$ induces a natural isomorphism of $H^{2}(G, \mathbb{T})$ onto $H^{2}(S, \mathbb{T})$.

Corollary 3.5. In the situation of Theorem 3.2, the original $\sigma$-representation of $S$ can be dilated to a unitary $\rho$-representation of $G$, where $\rho$ is any Borel multiplier extending $\sigma$.

\section{REFERENCES}

[Arv] W. B. Arveson, An addition formula for the index of semigroups of endomorphisms of $\mathscr{B}(\mathscr{H})$, Pacific J. Math. 137 (1989), 19-36.

[C-P] A. H. Clifford and G. B. Preston, The algebraic theory of semigroups, Vol. I, Math. Surveys Monographs, vol. 7, Amer. Math. Soc., Providence, RI, 1961.

[Che] P. R. Chernoff, Extensions and triviality of multipliers on subsemigroups of the reals, Semigroup Forum 41 (1990), 237-244.

[Din] H. Dinh, Multipliers on subsemigroups of the real line, Proc. Amer. Math. Soc. 117 (1993), 783-788.

[Dou] R. G. Douglas, On extending commutative semigroups of isometries, Bull. London Math. Soc. 1 (1969), 157-159.

[P-R] J. Phillips and I. Raeburn, Semigroups of isometries, Toeplitz algebras and twisted crossed products, Integral Equations Operator Theory 17 (1993), 597-602.

Department of Mathematics, University of Newcastle, New South Wales 2308, AusTRALIA

E-mail address: marceloefrey.newcastle.edu.au

E-mail address: iain@frey.newcastle.edu.au 